\title{
COVID-19 Pandemic Increased Anxiety Among Patients with Inflammatory Bowel Disease: A Patient Survey in a Tertiary Referral Center
}

\author{
Molly L. Stone ${ }^{1}$ (D) Minjun Feng ${ }^{2} \cdot$ Erin M. Forster $^{2}$
}

Received: 22 April 2021 / Accepted: 2 June 2021 / Published online: 11 June 2021

(c) The Author(s), under exclusive licence to Springer Science+Business Media, LLC, part of Springer Nature 2021

\begin{abstract}
Background COVID-19 is the first global pandemic in more than 100 years, and at its onset, the effects were largely unknown. Immunocompromised patients, including IBD, were presumed to have higher risk.

Aims We hypothesized patients with IBD would have higher-than-baseline anxiety, high perceived vulnerability and significant lifestyle impacts as a result of the pandemic. We sought to assess the impact of these changes on disease and management.

Methods A cross-sectional study of patients with Crohn's disease, ulcerative colitis and IBD-unspecified was conducted. Patients were invited to participate by email in an IRB-approved brief, voluntary survey. Survey questions focused on disease characteristics, healthcare access and self-reported psychological well-being.

Results Responses from $492(\mathrm{CD}=337, \mathrm{UC}=141, \mathrm{IC}=14)$ patients were included in the analysis. The majority of patients with IBD had increased anxiety since the pandemic, which correlated with an increase in GI symptoms. This risk of symptoms was mitigated by communication with their provider. Many patients had lifestyle changes including requesting time off work due to perceived vulnerability and changes in eating habits.

Conclusions Our findings support an increase in illness-associated anxiety and perceived vulnerability among patients with IBD during the COVID-19 pandemic. Open communication with providers is important to maintain adequate control of disease and reduce symptoms of flares triggered by ongoing stress.
\end{abstract}

Keywords Inflammatory bowel disease · Anxiety $\cdot$ COVID-19 $\cdot$ Perceived vulnerability

\section{Introduction}

On March 11th 2020, the World Health Organization declared COVID-19, the disease caused by the novel virus SARS-CoV-2, a pandemic. This incited worldwide fears about the impact of the disease. During the first six months of the pandemic, it claimed 192,448 lives in the United States with mortality rates as high as $9.5 \%$ during the height of the outbreak [1,2]. Immunocompromised individuals and

Molly L. Stone

stonemo@musc.edu

1 Department of Internal Medicine, Medical University of South Carolina, 96 Jonathan Lucas Stree, Suite 812, Charleston, SC 29425, USA

2 Division of Gastroenterology and Hepatology, Department of Internal Medicine, Medical University of South Carolina, Charleston, SC, USA those with comorbidities have been identified as high risk for contracting disease as well as having higher morbidity and mortality [3]. Patients with inflammatory bowel disease (IBD) have been presumed to fall into this high risk category due disease characteristics as well as immunomodulatory medications used to control disease, although this has not yet been supported by evidence thus far $[4,5]$.

IBD providers have adapted to utilizing more telehealth services in order to decrease spread of illness, which has resulted in up to $90 \%$ of visits being successfully transitioned to virtual interaction with high levels of patient satisfaction in surveys across the country [6-8]. Many patients have been reticent to continue their maintenance therapy during the pandemic with nearly one-third of patients missing doses, citing concerns about visiting healthcare facilities to acquire the medication or worries that it would place them at increased risk for contracting it [9]. Lapses in therapy 
have widely been associated with flares, development of antibodies, and need for IBD-related surgery [10-11].

In surveys of patients with IBD across the world, most had an increase in illness-related anxiety from baseline and perceived increased vulnerability due to disease $[8,9,12$, 13]. At baseline, patients with IBD have higher rates of anxiety and depression compared to the general population [14]. In response to the pandemic, anxiety rates in patients with IBD have been high as $48-62 \%[9,15]$. Perceived stress is strongly correlated with increasing GI symptoms regardless of the cause of the stress [16]. Patients do find some relief of their worry by talking to their providers, though this effect was small in an Italian survey [17].

We hypothesized patients with IBD living in the United States would have increased levels of anxiety and perceived vulnerability to COVID-19. Understanding patients' perception of their risk and effects on their lifestyle is imperative in continuing to provide care as the pandemic continues through the coming months.

\section{Methods}

\section{Study Design}

A cross-sectional study was conducted between June 15 and July 5, 2020 of patients with IBD who had been seen at the Medical University of South Carolina in the previous five year period. This was approved by the Institutional Review Board (IRB) prior to initiation of the project. The IRB completed a full ethical evaluation of the project including review of survey questions prior to distribution to the patient population. All participants provided informed consent for participation in this study.

All patients who had been seen at the IBD center with a diagnosis of Crohn's disease (CD), ulcerative colitis (UC) or IBD-unspecified (IBDU) and had an email address listed in their electronic medical record (EMR) were included in the study. A link to the informed consent and survey was sent to the single email address listed in the demographics section of the EMR to all patients simultaneously using REDCap secure data collection software. Patients received weekly email reminders to complete the survey during the 21 day data collection period. Once patients submitted, their responses were recorded and they were unable to return to the survey. The survey closed to additional responses at the conclusion of the study period. All patients were $>18$ years old at the time of participation. A total of 1504 patients were invited to participate.

\section{Data Acquisition}

Patients were invited by email to participate in an anonymous survey of questions related to their disease, healthcare access, mental and physical well-being during this time. Questions assessed disease characteristics, access to care, eating habits, reactions to the pandemic and mental well-being during the pandemic period prior to the time of the survey, see full survey in Online appendix 1. Disease assessment including disease activity, changes in symptoms, current medications and recent changes in therapy. Access was assessed with questions related to insurance coverage, willingness to have labs/procedures, ability to access their healthcare provider (HCP) and discuss concerns as well as feeling of support. Eating habits were assessed for absolute changes as well as free-text commentary detailing types of changes. Pandemic reactions included use of masks and sources of information related to the pandemic. Mental health questions focused on perceived vulnerability and anxiety related to illness.

\section{Data Management}

All responses were recorded in a secure database system, REDCap and data were analyzed using Microsoft Excel. Statistical analysis was performed using Excel. Chi-square analysis was used to compare means with statistical significance set at $p<0.05$.

\section{Results}

\section{Patient and Disease Characteristics}

A total of 509 patients completed the survey, 17 responses by patients without an IBD diagnosis were excluded, and a total of $492(\mathrm{CD}=337, \mathrm{UC}=141, \mathrm{IC}=14)$ were included in analysis. Patients were excluded if they chose "other" and entered a non-IBD diagnosis into the freetext response (ex. Microscopic colitis, radiation colitis, primary sclerosing cholangitis, irritable bowel syndrome, etc.) (Table 1).

The majority of patients ( $72 \% \mathrm{CD}, 63 \% \mathrm{UC}, 79 \%$ IC) endorsed feeling more anxious since the beginning of the pandemic. Patients who were anxious about the situation were more likely to experience symptom worsening ( $32 \%$ vs $7 \%, \mathrm{~N}=492, p<0.05)$. Of those with worsen symptoms reported $(\mathrm{N}=121)$, the most commonly reported was increased frequency of bowel movements $(\mathrm{N}=96)$ followed by worsening pain $(\mathrm{N}=89)$, few patients experienced more bleeding $(\mathrm{N}=37)$. 
Table 1 Patient demographics and characteristics

\begin{tabular}{ll}
\hline $\begin{array}{l}\text { Diagnosis }(N=492) \\
\text { Crohn's disease }\end{array}$ & $337(68 \%)$ \\
Ulcerative colitis & $141(29 \%)$ \\
Indeterminate colitis & $14(3 \%)$ \\
Highest Level of Education $(N=482)$ & \\
Less than high school & $2(<1 \%)$ \\
High school & $128(27 \%)$ \\
Associate Degree & $90(19 \%)$ \\
Bachelors' Degree & $148(31 \%)$ \\
Masters Degree & $87(17 \%)$ \\
Doctorate & $30(6 \%)$ \\
Reliable internel acess & $485(99 \%)$ \\
Own a smat phone & $473(96 \%)$ \\
Loss of health insurance & $3(<1 \%)$ \\
Current medication $(N=492)$ & \\
No therapy & $41(8 \%)$ \\
Steroid monotheraphy & $7(1 \%)$ \\
5-ASA monotheraphy & $47(10 \%)$ \\
Immunomodulator monotheraphy & $19(4 \%)$ \\
Biologic monotheraphy & $202(41 \%)$ \\
Dual theraphy $(N=125)$ & \\
Steroid + ASA & $6(5 \%)$ \\
Steroid + Biologic & $14(11 \%)$ \\
ASA + Immunomodulator & $10(8 \%)$ \\
ASA + Biologic & $15(12 \%)$ \\
\hline &
\end{tabular}

Of those with worsening symptoms, only approximately half (49\%) discussed their symptoms with their HCP. Additionally, many ( $79 \% \mathrm{CD}, 68 \% \mathrm{UC}, 72 \%$ IC) patients were worried and they were more vulnerable to COVID-19 infection, but less than half ( $54 \% \mathrm{CD}, 42 \% \mathrm{UC}, 33 \%$ IC) discussed their concerns with their HCP. Patients who discussed their concerns had a higher rate of feeling supported compared to those who did not ( $92 \%$ vs $70 \%, \mathrm{~N}=487, p<0.05$ ). Feeling of support from their HCP was also associated with lower rates of symptom worsening. Specifically, patients who felt supported had a lower rate of developing worsening of IBD symptoms compared to those who felt unsupported (22\% vs $31 \%, \mathrm{~N}=471, p<0.05)$. Only $6 \%(\mathrm{~N}=30)$ had an increased dose of a current medication $(\mathrm{N}=19)$ or new medication added $(\mathrm{N}=11)$ during the course of the pandemic, all of these patients had discussed their symptoms worsening with their HCP. Response to medication changes made during the pandemic with symptomatic and endoscopic assessment was not included in this single time-point survey.

\section{Communication and Lifestyle Changes}

Forty-six percent $(\mathrm{N}=227)$ of patients communicated with their IBD provider during the pandemic. Of those in
Table 2 Patient reported comfort with comman procedures (N-492)

Procedure

Having labs drawn

$73 \%(\mathrm{~N}-361)$

Provideing a stool smaple

$55 \%(\mathrm{~N}-271)$

Having imaging (CT, MRI, ultrasound)

$60 \%(\mathrm{~N}-294)$

Going to infusion center

$52 \%(\mathrm{~N}-257)$

Having a colonoscopy

$50 \%(\mathrm{~N}-247)$

Not comfortable with any procedure

$21 \%(\mathrm{~N}-103)$

communication, the most common methods were through the EMR messaging function (46\%) and by phone (23\%). Eleven patients endorsed the desire for more information related to how the pandemic affected those with IBD in the free-text comments. Patients were often uncomfortable accessing healthcare facilities for procedures or therapies (Table 2). Patients used an average of 1.77 of the listed resources (websites, news, social media, other), with most patients getting information from the news $(\mathrm{N}=366)$ and websites $(\mathrm{N}=333)$. Commonly reported websites were the Centers for Disease Control $(\mathrm{N}=119)$, Department of Health and Environmental Control $(\mathrm{N}=64)$, and the Crohn's and Colitis Foundation $(\mathrm{N}=8)$.

Patients with IBD had significant changes to their lifestyle during the pandemic. Three patients $(<1 \%)$ lost insurance coverage as a result of unemployment caused by the pandemic. Seven percent $(\mathrm{N}=32)$ of patients requested a work excuse due to perceived vulnerability related to their IBD with $59 \%(\mathrm{~N}=19)$ requesting paperwork from their IBD provider. Those with perceived vulnerability were also significantly more likely to use a mask in public areas $(p<0.01)$. The most common type of mask used was homemade (47\%, $\mathrm{N}=233)$ followed by surgical masks $(44 \% \mathrm{~N}=217)$. Less than $15 \%$ of all patients with IBD (10\% CD, $7 \%$ UC, $14 \%$ IBDU) reported not using a mask at all in public places.

Thirty percent of patients endorsed changes in their eating patterns during the pandemic. Of those, $95 \%(\mathrm{~N}=142)$ provided free-text responses with nearly half (47\%) endorsing "healthier" changes such as eating at home, cooking their own meals, increasing fruit and vegetable intake. Only $13 \%$ felt their habits had worsened, the remaining $40 \%$ were neutral changes. There was not a significant association between worsened eating habits and symptom flare ( $24 \%$ vs $28 \%$, $\mathrm{N}=89, p 0.36$ ).

\section{Conclusions}

This study supports an increase in illness-associated anxiety among patients with IBD during the global pandemic caused by COVID-19. Our population had high levels of perceived vulnerability, similar to other surveys of worldwide, which 
increased their illness-related anxiety $[9,15]$. Contrary to Italian patients at the height of their outbreak, patients in the United States that felt supported had lower rates of symptom worsening if they discussed their concerns surrounding the pandemic with their HCP. This highlights the importance of regular availability of HCP to communicate with their patients. Forty-six percent of our patients who discussed concerns with their HCP utilized the EMR secure messaging feature which allows convenient communication with the burden of response shared among multiple providers [18]. In the open commentary section, many patients indicated a desire for more readily available information about their risks and recommendations from their GI specialist. Patients were primarily getting their information about the pandemic from websites and news media (67\% and $74 \%$, respectively) compared to social media (24\%). Of the websites reported, the most common was the Centers for Disease Control which is a reputable source.

The majority of patients felt their IBD made them more vulnerable to COVID-19, and often impacted their daily activities. More than $85 \%$ of patients reported wearing a mask (homemade, surgical/N95 or bandana) with a significant association between feeling vulnerable and use of a mask $(p<0.01)$. With universal masking and the protocols in place at our institution, only $73 \%$ of patients were comfortable coming in-person for lab studies and only half were comfortable presenting for stool studies or colonoscopy (55\% and 50\% respectively). With less objective data available (when compared to in-person visits), it can be challenging for providers to assess and react to changes in patients' symptoms. Though only six percent of patients in our cohort required addition of a new medication or an increase in dose of a current therapy, this may be under-represented due to lack of objective data as one quarter (24\%) had increased symptoms since the beginning of the pandemic and $62 \%$ of those discussed changes with their HCP (Fig. 1).

Patients endorsed a variety of lifestyle changes during the pandemic period including 30\% with changes to their eating habits. Though worse eating habits were not associated with increased IBD symptoms in our limited time period, historical evidence suggests heavily processed foods do promote gut inflammation and worsening symptoms [19]. Four patients also reported increasing their physical activity due to spending more time at home and none of these patients experienced symptom worsening, which is consistent with established data that physical activity improves quality of life for patients with IBD [20]. Overall, there was a variety of responses; those with increased anxiety also reported more detrimental changes to their lifestyle including poorer diet, more alcohol intake and less exercise which highlights the important interplay of mental and physical well-being in this population.

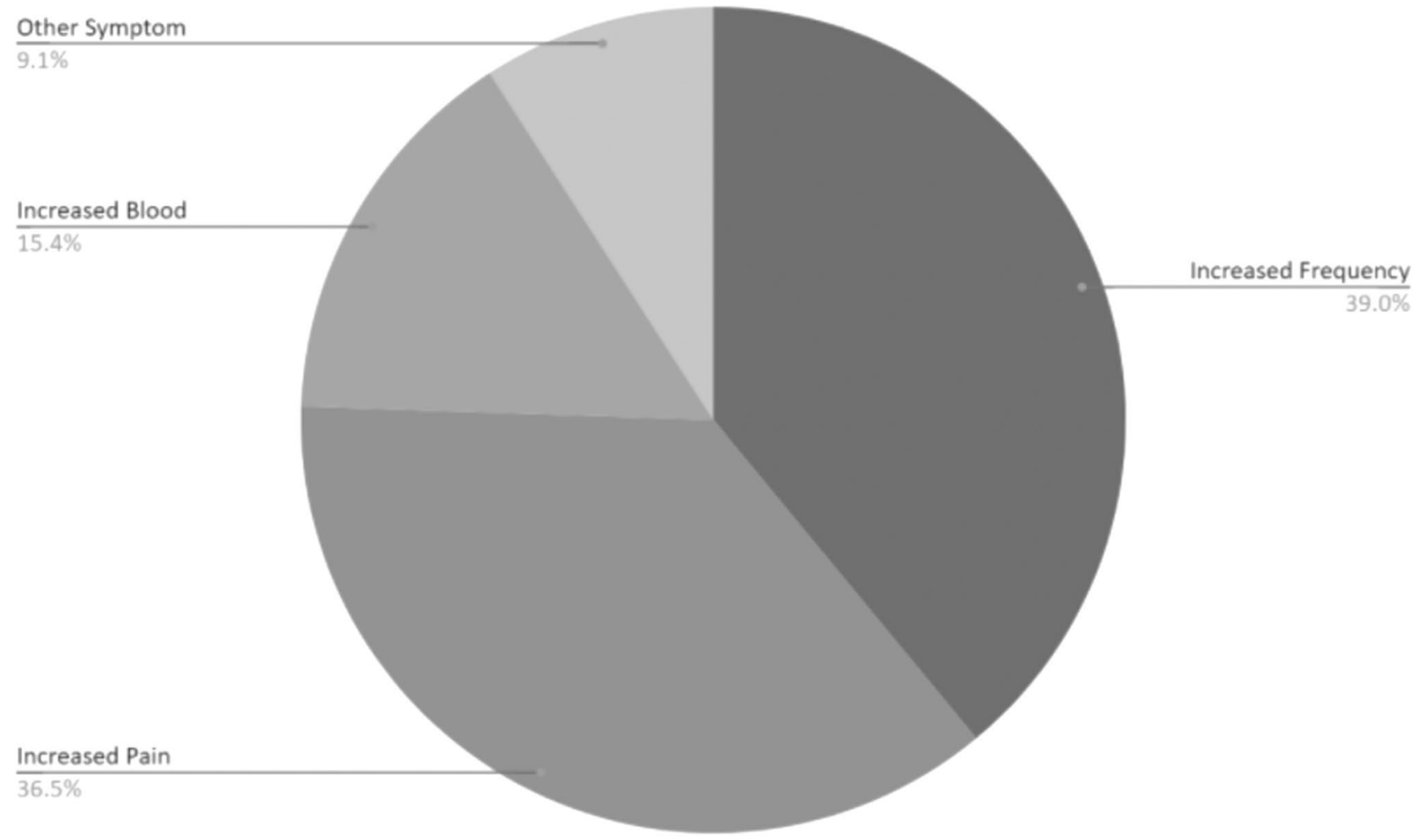

Fig. 1 Breakdown of reported symptoms 
This is the first study looking at the psychological impacts of COVID-19 on patients with IBD in the United States which has the highest number of COVID-19 deaths in the world [1]. Our study has several additional strengths including the wide catchment of a tertiary referral center with associated variety in patient diagnosis and complexity. Limitations of this study include the sample size and subjective nature of selfreport without any clinical corollary. Only $34 \%$ of patients who received the survey completed responses, which is overall approximately $20 \%$ of all patients with IBD in our system. Diagnosis and medications were reported by patients, previous studies have noted that only approximately $60 \%$ of patients understand the role of their therapy [21]. We are also limited by the web-based distribution of the study which excludes patients who do not have regular internet access. Our sample was limited to patients with an email address listed in their electronic medical record; the general population in the catchment area has internet access in only $76 \%$ of homes [22]. The self-selection of the population may also influence the willingness of patients included in the study to utilize technology to communicate with their providers in a timely fashion about changes to symptoms and therefore earlier intervention.

The impact of this global pandemic will surely be felt for decades to come, longer-term studies are needed to assess the impact of missed and delayed procedures during this period on long-term disease control. As social isolation continues, there will also be a role for reassessment of the psychological impact in patients with IBD as they continue to remain distant from both loved-ones and reduced healthcare access. Future studies could also address how patients with IBD without regular access to the internet and telehealth visits/communication have coped during this time period.

Supplementary Information The online version contains supplementary material available at https://doi.org/10.1007/s10620-021-07095-y.

Author's contribution M.S. data review and first draft of manuscript, M.F. study design, distribution to subjects and data compilation, E.F. project background, study design, and manuscript review.

\section{Funding None.}

Data availability The data underlying this article will be shared on reasonable request to the corresponding author.

\section{Declarations}

Conflict of interest None of the authors have a conflict of interest or disclosures.

\section{References}

1. NCHS Mortality Reporting System. Pneumonia, Influenza, and COVID-19 Mortality. CDC.gov.
2. Nuzzo J, Moss B, Khan J et al. Coronavirus Resource Center.

3. Luo L, Fu M, Li Y et al. The potential association between common comorbidities and severity and mortality of coronavirus 2019: A pooled analysis. Clinical Cardiology. Online ahead of print. https://doi.org/10.1002/clc.23465.

4. Monteleone G, Ardizzone S. Are patients with Inflammatory Bowel Disease at Increased Risk for COVID-19 Infection. Journal of Crohn's and Colitis. 2020. 1-3.

5. Aziz M, Fatima R, Haghbin H, Lee-Smith $W$ and Nawras A. The Incidence and Outcomes of COIVD-19 in IBD Patients: A Rapid Review and Meta-analysis.Inflammatory Bowel Disease; 2020:26 (10):e132-e133.

6. Taxonera C, Alba C, Olivares D, Martin M, Ventero A and Canas M. Innovation in IBD Care During the COVID-19 Pandemic: Results of a Cross-Sectional Survey on Patient-Reported Experience Measures. Inflammatory Bowel Disease; 2020.

7. Garrido I, Lopes S and Macedo G. Inflammatory Bowel Disease Management in a Major Referral Center During COVID-19 Pandemic. Inflammatory Bowel Disease; 2020.

8. Gomes C, Pinho R, Ponte A, Silva JC, Afecto E, Correia J and Carvalho J. Patient's perspective on the implementation of measures to contain the SARS-CoV-2 pandemic in a Portuguese Gastroenterology Department. European Journal of Gastroenterology and Hepatology;2020:33(4):527-532.

9. Mosli, Alourfi, Alamoudi et al. A cross-sectional survey on the psychological impact of the COVID-19 pandemic on inflammatory bowel disease patients in Saudi Arabia. Saudi Journal of Gastroenterology; 2020:26(5)263-271.

10. Van der Have M, Oldenburg B, Kaptein AA, et al. Non-adherence to Anti-TNF Therapy is Associated with Illness Perceptions and Clinical Outcomes in Outpatients with Inflammatory Bowel Disease: Results from a Prospective Multicentre Study. Journal of Crohn's and Colitis. 2016; 10(5):549-555.

11. Govani SM, Noureldin M, Higgins PD, et al. Defining an Optimal Adherence Threshold for Patients Taking Subcutaneous Anti-TNFs for Inflammatory Bowel Diseases. American Journal of Gastroenterology. 2018; 113(2)276-282.

12. Castellini G, Savarese M, Previtali E, Armuzzi A, and Graffigna G. Italian IBD Patients Coping With COIVD-19 Emergency: The Mitigating Role of Psychological Readiness to Engage in Selfcare. Inflammatory Bowel Disease. 2020.

13. Schlabitz F, Teich N, Michl P and Walldorf J. Inflammatory Bowel Disease and COVID-19--'Preventive" Sick Certificated as a Frequent Coping Strategy in the Face of the Pandemic. Inflammatory Bowel Disease. 2020.

14. Qualter P, Rouncefield-Swales A, Bray L. Depression, anxiety and loneliness among adolescents and young adults with IBD in the UK: the role of disease severity, age of onset and embarassment of the condition. Quality of Life Research. 2020; 30(2):497-506.

15. Spagnuolo R, Larussa T, Iannelli C, et al. COVID-19 and Inflammatory Bowel Disease: Patient Knowledge and Perceptions in a Single Center Survey. Medicina. 2020; 56(407):1-11.

16. Targownik LE, Sexton KA, Bernstein MT, Beatie B, Sargent M, Walker JR and Graff LA. The Relationship Among Perceived Stress, Symptoms and Inflammation in Persons with Inflammatory Bowel Disease. American Journal of Gastroenterology. 2015;110(7):1001-12.

17. D'Amico F, Rahier JF, Leone S, Peyrin-Biroulet L and Danese $\mathrm{S}$. Views of patients with inflammatory bowel disease on the COVID-19 pandemic: a global survey. The Lancet Gastroenterology and Hepatology. 2020; 5:631-32.

18. North F, Luhman KE, Mallmann EA, Mallman TJ, TulledgeScheitel SM, North EJ and Pecina JL. A Retrospective Analysis of Provider-to-Patient Secure Messages: How Much are they Increasing, Who is doing the Work and is the Work happening after hours?JMIR Medical Informatics. 2020; 8(7):e16521. 
19. Marion-Letellier R, Amamou A, Savoye G, Ghosh S. Inflammatory Bowel Diseases and Food Additives: To Add Fuel on the Flames. Nutrients. 2019;11(5)1111.

20. Packer N, Hoffman-Goetz L, Ward G. Does physical activity affect quality of life, symptoms and immune measures in patients with inflammatory bowel disease? A systematic review.Journal of Sports Medicine and Physical Fitness. 2010; 50(1)1-18.

21. Linn AJ, van Dijk L, Smit EG, Jansen J, van Weert JC. May you never forget what is worth remembering: the relation between recall of medical information and medication adherence in patients with inflammatory bowel disease. Journal of Crohn's and Colitis. 2013; 7(11):543-50.

22. United States Census Bureau. Quick Facts.2019. census.gov/ quickfacts.

Publisher's Note Springer Nature remains neutral with regard to jurisdictional claims in published maps and institutional affiliations. 